\title{
Selection for changes in the incidence of wing dimorphism in Gryllus firmus
}

\author{
D. A. Roff
}

\author{
Department of Biology, McGill University, \\ 1205 Dr Penfield Avenue, Montreal, \\ Quebec, Canada, H3A 1B1.
}

Previous studies suggested that in the cricket Gryllus firmus the heritability of wing dimorphism is approximately $0 \cdot 65$. This estimate was based on a full sib analysis and hence may be confounded by non additive effects. To confirm this estimate a selection experiment was undertaken, lines being selected for increased and decreased incidence of macroptery. The response to selection was asymmetric, response for decreased percentage macroptery being faster than that for increased proportion macroptery. The realized heritability in the "up" line was approximately $0 \cdot 25$, and in the "down" line approximately 0.95 , the mean across all lines being 0.6 : this agrees very well with the estimate of 0.67 obtained from a full sib analysis. Two possible reasons for the asymmetric response are suggested. In the related cricket species, Gryllus rubens, the genes controlling wing morph appear to be on the sex chromosome while in $G$. firmus no sex linkage is evident.

A new method of estimating realised heritability for threshold traits is presented.

\section{INTRODUCTION}

Migration is an important component of the lives of many insects permitting them to colonize and persist in temporary habitats (Southwood, 1962; Johnson, 1969; Harrison, 1980; Dingle, 1985). But though there are long term benefits to migration there are also costs such as increased mortality and decreased reproduction (Southwood, 1962; Roff, 1977), and hence it is not surprising that migratory polymorphisms are common (Harrison, 1980; Dingle, 1985). Many insect species are wing dimorphic, some individuals within the population or a family having wings and being capable of flight and others having either no wings or reduced wings and being incapable of flight. Within these species there is a clear dichotomy between the morph capable of migration and that which is not. But an individual with wings does not have to fly and hence the existence of wing dimorphic species suggests that there is a cost not only to migration itself but also to possessing the capability of migration, viz wings, wing muscles and associated structures. This hypothesis has been confirmed in a wide variety of insects, in which it has been found that the flight-capable morphs show a delay in the onset of reproduction and a reduction in fecundity (Roff, 1984, 1986a; Denno et al., 1989; Roff and Fairbairn, 1990). Thus there is a trade-off between flight capability and reproduction.

To understand how the incidence of a particular wing morph will evolve under changing environmental conditions we must understand the genetic basis of the traits involved in this trade-off and the genetic correlations between them. Heritability estimates of wing dimorphism for two crickets, Gryllus firmus and Allonemobius fasciatus, suggest that a significant fraction of the variation (>50 per cent) can be attributed to additive genetic effects (Roff, 1986 b; Mousseau and Roff, 1989; maternal effects were insignificant in the study of G. firmus but could not be examined in the analysis of $\boldsymbol{A}$. fasciatus). However, in both cases heritability estimates were obtained by full sib analysis and thus contain an unknown fraction of non additive effects. To overcome this deficiency a selection experiment was undertaken using Gryllus firmus, lines being selected for increased and decreased incidence of macroptery. The results of this experiment and a new method for estimating realized heritability of a threshold trait are presented in this paper. 


\section{MATERIALS AND METHODS}

\section{Experimental protocol}

Details of the species and rearing methods are given in Roff (1986b), and only the salient points are presented here. The stock of G. firmus used in the present study was derived from approximately 40 individuals (approximate sex ratio $1: 1)$ from a single location in northern Florida in 1981. They are maintained in diapause averting conditions $\left(25-30^{\circ} \mathrm{C}\right.$, no set photoperiod but the laboratory lights ensure a relatively long light period), with a breeding stock of between 100-300 individuals. For the selection experiments individuals were raised in batches of 60 individuals per disposable mouse cage, as described in Roff $(1986 b)$. Food was provided, ad libitum and comprised Purina ${ }^{\circledR}$ rabbit chow and fresh lettuce leaves.

Previous studies were conducted at $30^{\circ} \mathrm{C}$ and a photoperiod of $17 \mathrm{~h} \mathrm{~L} ; 7 \mathrm{~h} \mathrm{D}$ : under these conditions the proportion of macropterous males and females is about 76 and 64 per cent, respectively (Roff, 1986b). To reduce this percentage to around 50 per cent in the females, in the present experimen1 crickets were reared at $28^{\circ} \mathrm{C}, 15 \mathrm{~h}$ L: $9 \mathrm{~h} \mathrm{D}$. An estimate of the heritability of wing dimorphism under these conditions was obtained in the first selection experiment as follows: eggs were obtained from the stock culture and the nymphs raised under the experimental conditions. From this group 20 pairs were extracted comprising 10 pairs $\mathrm{LW} \times \mathrm{LW}$ (macropterous $\times$ macropterous) and 10 pairs $\mathrm{SW} \times \mathrm{SW}$ (micropterous $\times$ micropterous). Estimates of the heritability of wing dimorphism were made from these crosses for the founding population. Since these matings do not include mixed crosses, estimates of heritability have to be corrected for assortative mating (discussed below).

To establish a macropterous line (hereafter referred to as the L1 line) 200 adults ( 100 males, 100 females) from the $10 \mathrm{LW} \times \mathrm{LW}$ matings were mixed together, with approximately equal representation from each family. Similarly, a micropterous line (S1) was started by mixing together the offspring from the $\mathrm{SW} \times \mathrm{SW}$ crosses. A Control line (C1) was formed by mixing the offspring from all crosses. Based on initial estimates of percentage macroptery in each family, 3 "extreme" families were selected and matings made between siblings within each family, 20 females and ten males per family: final data on these families indicated that they were not "extreme" (see Results) but the lines were maintained to provide further estimates of heritability. One "within family" macropterous and two micropterous lines were so established, designated as WL1, WS1 and WS2, respectively.

For each of the lines L1, S1 and C1, six cages containing 60 newly hatched nymphs per cage, and for the within family lines (WL1, WS1 and WS2), two cages of 60 nymphs/cage were established. Males and females of a desired morph (macropterous for L1, micropterous for S1 and both for $\mathrm{C1}$ ), were selected upon eclosion into adults until approximately 100 (and never less than 50 ) of each sex were obtained. In the case of WL1, WS1 and WS2, 20 females and ten males were selected. The relatively large number of parents was used to prevent inbreeding depression. The mass selection procedure outlined above was followed on all generations subsequent to the first. To provide a replicate, a second series of lines was set up some months after the first, the protocol followed being identical except that a mass selection design was followed from the outset and no initial group of single pair matings constructed. These lines will be designated L2, S2 and C2.

In $G$. rubens wing morph is controlled to a large extent by sex-linked genes (Walker, 1987; Gryllus have an XO system with the female being $\mathrm{XX}$ ); to test for this possibility in $G$. firmus, the following crosses were made in generation 10 of the first experiment, $\mathrm{L} 1 \times \mathrm{S} 1$, $\mathrm{L} 1 \times \mathrm{WS} 1, \mathrm{~L} 1 \times \mathrm{WS} 2, \mathrm{WL} 1 \times \mathrm{S} 1, \mathrm{WL} 1 \times \mathrm{WS} 2 . \mathrm{In}$ each cross two cages, each comprising ten adult females from one line and five adult males from another, were set up and 180 nymphs collected from these for rearing. All crosses were reciprocal with respect to sex.

\section{Statistical analysis}

The estimation of heritability from full sib data for a threshold trait is summarised in Roff (1986b, typographical corrections to formulae given in this paper are presented in Mousseau and Roff, 1989). In the present analysis the situation is complicated by the fact that the matings are assortative. To calculate the uncorrected estimate of heritability, $H$, we require an estimate of the mean proportion of micropterous individuals per family, $p$. The proportion $p$ is normally estimated by $(1 / n) \sum p_{i}$, where $n$ is the number of families and $p_{i}$ is the proportion of micropterous individuals in family $i$ (Roff, $1986 b)$. Since in the present case mating is assortative, $p$ was estimated from the parental population. The heritability estimate can be corrected using 
the formula given by Falconer (1981, p. 164),

$$
\hat{h}^{2}=\{-1+\sqrt{[1+4 r H]}\} /(2 r)
$$

where $\hat{h}^{2}$ is the narrow sense heritability, $H$ is the uncorrected estimate of heritability, and $r$ is the phenotypic correlation between parents measured on the underlying continuous scale. This cannot be directly estimated from the dichotomous phenotypes of the parents (macropterous or micropterous). However, given the proportions of macropterous males and females in the population from which the parents are derived, and assuming that the dichotomous trait is a consequence of an interaction between a threshold and a continuously varying character (Falconer, 1981), $r$ can be estimated by Monte Carlo simulation. The base population comprised 51 per cent macropterous females and 29 per cent macropterous males. The value of $r$, estimated from 100 runs of the simulation was 0.63 . To examine the robustness of this value to variation in percentage macroptery I ran the simulation with percentages of macropterous males and females ranging from 20-50 per cent, using both equal and unequal values for the parents. The mean value of $r$, estimated from 100 runs per parameter combination, was very insensitive to variation within this range, the maximum and minimum values being 0.66 and 0.62 , respectively.

Realized heritability is usually estimated by a regression of cumulative response on cumulative selection differential (Falconer, 1981). The same procedure can be used with a threshold trait in the following manner (Method 1): the mean value, on the underlying continuous scale, of the offspring in generation $i$ is estimated as $z_{i}$, the ordinate on the standardised normal curve corresponding to $p_{i}$ is estimated as $z_{i}$, the proportion of micropterous offspring. The estimated value of the micropterous parents selected to produce the $i+1$ generation is given by $z_{i}+\exp \left(-0 \cdot 5 z_{i}^{2}\right) /\left(\sqrt{[2 \pi]} p_{i}\right)$. For the macropterous line the relevant estimates are $-z_{i}$, and $-z_{i}-\exp \left(-0 \cdot 5 z_{i}^{2}\right) /\left(\sqrt{[2 \pi]}\left[1-p_{i}\right]\right)$.

A potential problem with the above method is that it cannot be used if in one or more generations only one morph is observed, since $z_{i}$ cannot then be estimated. This problem is likely to arise when samples are small and/or $p$ is close to 1 or 0 . A method that does not depend upon this constraint is as follows (Method 2): let $G_{\mathrm{s}, i}$ be the mean phenotypic value of the selected micropterous parents in the $i$ th generation. The expected value of the offspring, $z_{i+1}$, is given by

$$
z_{i+1}=z_{i}\left(1-h^{2}\right)+h^{2} G_{s, i} .
$$

The probability that an individual in generation $i+1$ will be micropterous is,

$(1 / \sqrt{[2 \pi]}) \int_{0}^{\infty} \exp \left(-0 \cdot 5\left(x-z_{i+1}\right)^{2}\right) d x=P_{i+1}$.

The likelihood of obtaining the observed series of micropterous individuals in the selection experiment is,

$$
\prod_{i=1}^{N} C_{r_{i}}^{n_{i}} P_{i}^{r_{i}}\left(1-P_{i}\right)^{n_{i}-r_{i}}
$$

where $n_{i}$ is the number of offspring in generation $i, r_{i}$ is the number of micropterous offspring in generation $i$, and $N$ is the number of generations. Taking logs, we obtain

$$
L L \propto \sum\left\{r_{i} \log \left(P_{i}\right)+\left(n_{i}-r_{i}\right) \log \left(1-P_{i}\right)\right\} .
$$

Thus the problem of finding the best estimate of $h^{2}$ reduces to finding the value of $h^{2}$ that maximizes LL. Apart from $h^{2}$, parameters to be estimated are the frequency of micropterous individuals in the initial population, and the mean phenotypic value of the parents at each generation. Although the frequency of macroptery differs between sexes, the two heritabilities do not differ (Roff, 1986 b), and hence in the present estimation procedure both sexes are considered jointly. The total number of parameters to be estimated is $2(N+1)+1$, where $N$ is the total number of generations. The best estimate of the initial frequency is $r_{0} / n_{0}$ (two estimates since there are two sexes), while the mean value of the parents can be computed according to the first method described, leaving only $h^{2}$ to be estimated. The best estimate of $h^{2}$, given the estimates of the other parameters as described above, can be found by numerical methods (see, for example, Press et al., 1986). Confidence intervals were estimated as the values of $h^{2}$ at which LL equals $\chi_{\alpha=0.05}^{2}$ with $2(N+1)+1$ degrees of freedom (Draper and Smith, 1981). Confidence intervals are asymmetric, but the differences were small and in the present paper the estimated standard error of the estimate was computed as $0 \cdot 5\left\{\left(C_{\mathrm{u}}-\hat{h}^{2}\right) / 2+\left(\hat{h}-C_{1}\right) / 2\right)$, where $C_{\mathrm{u}}$ and $C_{1}$ are, respectively, the upper and lower 95 per cent confidence bounds on $h^{2}$.

\section{RESULTS}

\section{Heritability estimates and selection response}

Heritability estimates derived from the 20 full sib families a re, after correction for assortative mating, 
$0.66 \pm 0.21( \pm$ S.E. $)$ and $0.69 \pm 0.22$ for females and males, respectively. These estimates compare very favorably to the values of $0.68 \pm 0.085$ and $0.62 \pm$ 0.075 for females and males, respectively, obtained under rearing conditions of $30^{\circ} \mathrm{C}$ and $17 \mathrm{~L}: 7 \mathrm{D}$ (Roff, 1986b). The relatively high value of $h^{2}$ suggests that the population should respond rapidly to selection. This prediction was verified by all selected lines, the lines selected for macroptery increasing to approximately 90 per cent macropterous females and those selected for microptery decreasing to approximately 5 per cent macropterous females by generation 5 (fig. 1). Based on ascending rank by percentage macropterous, the family selected as WL1 was 5th rank of the ten LW $\times$ LW crosses, while WS1 and WS2 were 3 rd and 5 th rank among the ten $\mathrm{SW} \times \mathrm{SW}$ crosses. The response of SW1 and WS2 were both more rapid than either S1 or S2, but the response of WL1 was comparable to L1 and L2 (fig. 1).

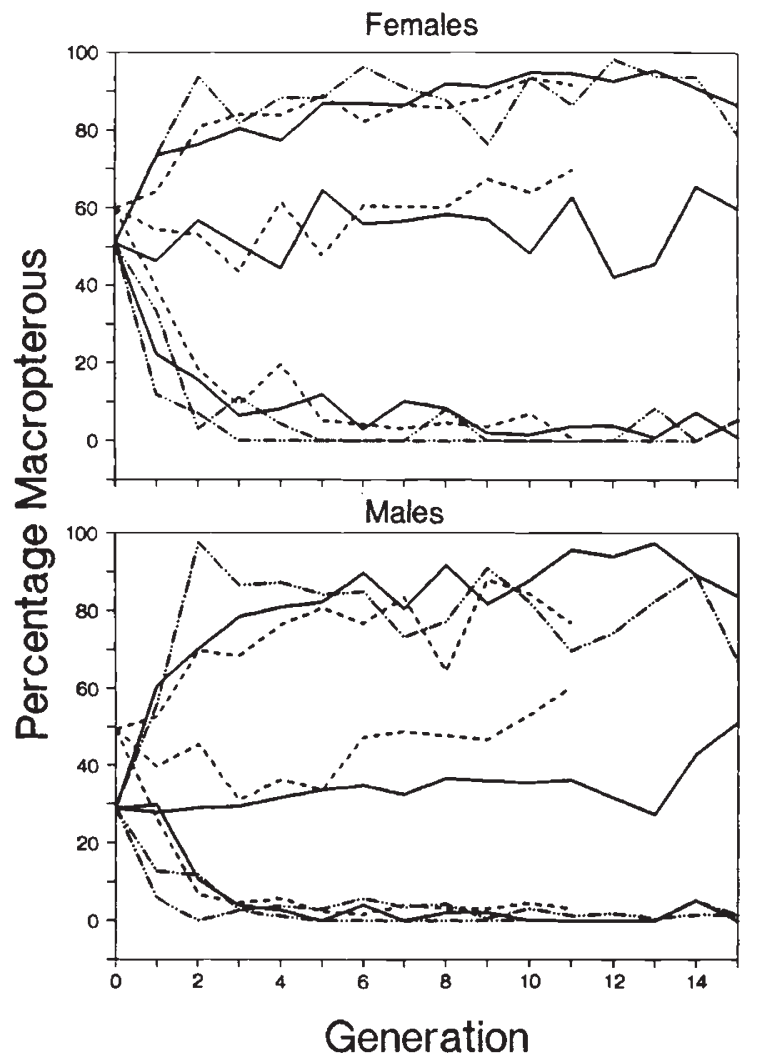

Figure 1 Response to selection for increasing and decreasing incidence of macroptery in Gryllus firmus. - Line 1 (L1, S1, C1);-·- - "Within family" lines (WL1, WS1, WS2); - - - Line 2 (L2, S2, C2).
The two methods of estimating $h^{2}$ from response to selection gave similar results and indicate that the realised $h^{2}$ in the macropterous lines was less than in the micropterous lines (table 1). In all three "within family" lines (WL1, WS1 and WS2) the estimated values of $h^{2}$ are similar in magnitude to the mass selected lines. Plots of cumulative response on cumulative selection differential were examined visually and statistically for evidence of curvature indicating a limit on the underlying scale to response to selection; there was no evidence of non linearity. The averaged estimate of heritability in the lines selected for increased percentage macroptery is $0 \cdot 25$, and that obtained for the lines selected for decreased incidence of macroptery is $0.95(0.90$ if estimates greater than 1 are set to 1$)$. The heritability estimate averaged across morphs and lines is 0.60 ; this agrees well with the value of 0.67 obtained from full sib analysis in the present experiment and that of 0.65 previously reported (Roff, 1984).

Table 1 Heritability estimates from selection experiments. See text for a description of the methods of estimation

\begin{tabular}{|c|c|c|}
\hline \multirow[b]{2}{*}{ Line } & \multicolumn{2}{|c|}{ Heritability estimate (S.E.) } \\
\hline & Method 1 & Method 2 \\
\hline L1 & $0.33(0.05)$ & $0.25(0.04)$ \\
\hline $\mathrm{L} 2$ & $0.22(0.05)$ & $0.33(0.04)$ \\
\hline WL1 & $0.11(0.10)$ & $0.24(0.07)$ \\
\hline S1 & $0.78(0.16)$ & $1.27(0.20)$ \\
\hline $\mathrm{S} 2$ & $0.86(0.18)$ & $0.74(0.11)$ \\
\hline WS1 & - & $0.72(0.19)$ \\
\hline WS2 & - & $1 \cdot 32(0 \cdot 32)$ \\
\hline $\mathrm{L} 1+\mathrm{S}_{1}{ }^{*}$ & $0.56(0.12)$ & $0.76(0.14)$ \\
\hline $\mathrm{L} 2+\mathrm{S} 2$ & $0.54(0.13)$ & $0.54(0.08)$ \\
\hline
\end{tabular}

* Mean of the two heritabilities. S.E. estimated as the square root of the mean variance.

\section{Crosses between lines}

There was no significant heterogeneity between cages within crosses, and therefore, cages were combined for the following analyses. If the genes controlling wing morph are sex linked, the frequency of macropterous offspring in highly selected lines should depend upon which parent is macropterous, as indicated in table 2. For $G$. rubens, crosses after five generations of selection indicated that a large proportion of the genes controlling wing morph are sex linked in this species (table 2; Walker, 1987). No such variation is 
Table 2 Percentage of macropterous offspring from crosses between lines selected for increased and decreased incidence of macroptery (LW). Sample sizes, within sexes, for the between line crosses ranged from 41 to 102 with a mean of $63(\mathrm{SD}=17)$

\begin{tabular}{lllc}
\hline $\begin{array}{l}\text { Female } \\
\text { parent }\end{array}$ & $\begin{array}{l}\text { Male } \\
\text { parent }\end{array}$ & $\begin{array}{l}\text { Female } \\
\text { offspring }\end{array}$ & $\begin{array}{l}\text { Male } \\
\text { offspring }\end{array}$ \\
\hline Macropterous* & Micropterous & $50.0(51)$ & $100.0(99)$ \\
Micropterous & Macropterous & $50.0(47)$ & $0.0(11)$ \\
L1 & L1 & 94.7 & 87.9 \\
WL1 & WL1 & 93.8 & 82.2 \\
S1 & S1 & 1.6 & 3.1 \\
WS1 & WS1 & 0.0 & 0.0 \\
WS2 & WS2 & 0.0 & 0.0 \\
L1 & S1 & 60.7 & 63.7 \\
S1 & L1 & 66.7 & 48.3 \\
WL1 & S1 & 47.2 & 16.7 \\
S1 & WL1 & 71.9 & 51.8 \\
L1 & WS1 & 75.6 & 72.9 \\
WS1 & L1 & 68.3 & 50.7 \\
WL1 & WS2 & 57.6 & 34.9 \\
WS2 & WL1 & 82.6 & 47.5 \\
L1 & WS2 & 79.6 & 70.0 \\
\hline
\end{tabular}

* The proportion expected if all genes controlling wing morph are sex linked and the lines homozygous. Values in brackets show data for $G$. rubens (from table 4 of Walker, 1987).

evident in any of the present crosses (table 2), indicating that in this species most of the genes controlling wing morph are autosomal.

\section{DISCUSSION}

Although the estimates of heritability derived from full sib analysis and from selection are very similar when morphs are combined (approximately 0.6, table 1), there is a clear asymmetry in the response to selection. Lines selected for increasing macroptery diverged from the control lines and approached their limiting value (100 per cent) much more slowly than lines selected for a decreased incidence of macroptery. Two factors may be responsible for this asymmetric response. First, two lines of evidence suggest that this may be a consequence, in part, of stress causing crickets to switch, developmentally, to the micropterous form;

(a) if crickets from the LW lines are weighed daily during development they all develop into the micropterous morph (Shannon and Roff, unpublished data).

(b) when reared under conditions that cause high mortalities ( $>50$ per cent) inordinately high frequencies of micropterous forms are obtained (50-100 per cent LW vs. 95 per cent
LW under "optimal" conditions; Roff, unpublished data).

Although the crickets in the selection experiments are reared under good conditions, as evidenced by high survival ( $>80$ per cent), some individuals are likely to be stressed due to small, random variations between and within cages, and possibly harassment by larger nymphs. Suppose that, if exposed to some stress, S, prior to the developmental stage at which wing morph is determined, a cricket will always develop into a SW morph. Further, suppose that, on average, 10 per cent of nymphs are exposed to $\mathrm{S}$ in each generation. Under these conditions lines selected for increasing macroptery will asymptote at 90 per cent rather than 100 per cent, while lines selected for decreased incidence of macroptery can achieve zero per cent. If, as is likely, genetic variation exists for the response to $\mathrm{S}$, selection for an increasing percentage of macroptery will also select against individuals that show a response to $\mathrm{S}$. Because of the very low selection intensity that can be achieved when the frequency of $\mathrm{LW}$ individuals exceeds 90 per cent (with respect to both the response to $S$ and the incidence of macroptery in the absence of S) the response to selection will be slow and the realized heritability relatively low, as found in the present experiments (fig. 1). However, the selected group in the line selected for increased microptery will contain some genetically macropterous individuals and hence the selection differential will be reduced. A slower response would therefore be expected in the $\mathrm{S}$ lines compared to the $\mathrm{L}$ lines although the former is capable of evolving further than the latter. The effect of $\mathrm{S}$ on the response to selection and the realized heritability is easily calculated using equations 2 and 3 and adjusting for the effect of $\mathrm{S}$. To demonstrate these effects I used $\mathrm{S}=0.1$ and a heritability of 0.8 in the absence of $\mathrm{S}$ (i.e., $\mathrm{S}=0$ ). In the case of selection for increased incidence of microptery neither the response to selection (fig. 2), nor the estimated realized heritability are significantly affected (the estimated value of $h^{2}$ declines from 0.8 to 0.76 ). But the response to selection when selection is for increased incidence of macroptery is greatly reduced (fig. 2), and the realized heritability only $0 \cdot 18$. These results match those observed (fig. 1) quite closely, but I have not attempted to estimate values of S or $h^{2}$ which give a "best" fit to the data.

The second possible reason for the difference in response is that micropterous females begin reproduction earlier and produce more eggs than the macropterous morph (Roff, 1984). It is possible that macropterous females with genotypes that lie 


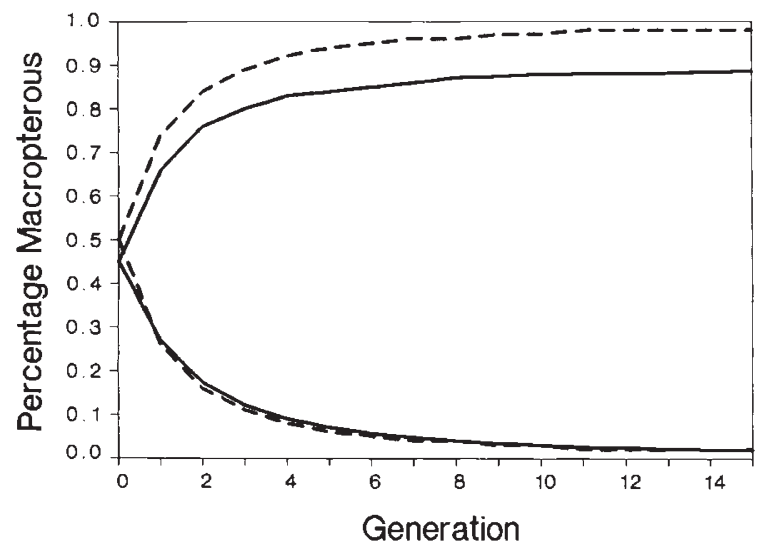

Figure 2 Predicted response to selection for increasing and decreasing incidence of macroptery when $h^{2}=0 \cdot 8, \mathrm{~S}=0$ $(---)$ and $\mathrm{S}=0 \cdot 1(-)$. In the latter case realized $h^{2}$ is reduced to 0.76 in the "down" line and 0.18 in the "up" line.

close to the threshold at which wing morph is determined also reproduce sooner and produce more eggs than macropterous females with genotypes that are far removed from the threshold. Since there is no way of distinguishing these genotypes morphologically both types would be selected but the former would contribute more offspring to the next generation. Likewise, in the micropterous females the age of first reproduction and total fecundity may depend upon how close the genotype is to the threshold value, micropterous females with genotypes far removed from the threshold reproducing the earliest and producing the most eggs. This would mean that natural selection would act against artificial selection when selecting for the macropterous morph but would act with artificial selection when selecting for the micropterous morph.

The finding that in two closely related species the genes controlling wing morph are in one case on the sex chromosomes ( $G$. rubens) and in the other on the autosomal chromosomes (G. firmus) was unexpected. Both species have the same number of chromosomes $(2 n=29$ in males, Lim et al., 1973; although $G$. firmus has not been examined, the species $G$. bermudensis, is now considered only a subspecies of $G$. firmus [Kevan, 1980]). Further study on other species within the genus Gryllus are needed to establish the general location of the genes determining wing morph.

Walker (1987) selected for changed incidence in the proportion of the macropterous morph of $G$. rubens, and as with $G$. firmus, obtained a rapid response, the realized heritability being $0.98 \pm 0.16$ (estimated by Method 1 using data presented in table 3 of Walker [1987]). The mechanism preserving such high genetic variation in these species has not been resolved though the trade-off between wing morph and the age schedules of reproduction (Roff, 1984) may play an important role.

Acknowledgements I am most grateful for the constructive criticisms of Drs D. J. Fairbairn, H. Dingle, J. Endler, T. Prout and M. Turelli. Catherine Helick and Sharon David provided indispensable technical assistence. This work was supported by an operating grant from National Science and Engineering Council of Canada.

\section{REFERENCES}

DENNO, R. F., OlmSteAd, K. L. AND McCloud, E. S. 1989. Reproductive cost of flight capability: a comparison of life history traits in wing dimorphic planthoppers. Ecol. Ent., $14,31-44$.

DINGLE, H. 1985. Migration. In Kerkut, G. A. and Gilbert, L. I. (eds) Comprehensive Insect Physiology, Biochemistry and Pharmacology. Vol. 9, Behaviour, Pergamon Press, New York.

DRAPER, N. R. AND SMITH H. 1981. Applied Regression Analysis, 2nd ed. John Wiley, New York.

FALCONER, D. S. 1981. Introduction to Quantitative Genetics. 2nd edn. Longman, London and New York.

HARRISON, R. G. 1980. Dispersal polymorphism in insects. Ann. Rev. Ecol. Syst., 11, 95-118.

JOHNSON, C. G. 1969. Migration and Dispersal of Insects by Flight. Methuen, London.

KEVAN, D. McE. 1980. The taxonomic status of the Bermuda beach cricket (Orthopteroida: Gryllidae). Syst. Ent., 5, $83-95$.

LIM, H.-C., VICKERY, V. R. AND McKEVAN, D. K. 1973. Cytogenetic studies in relation to taxonomy within the family Gryllidae (Orthoptera). 1. Subfamily Gryllinae. Can. J. Zool. 51, 179-186.

MOUSSEAU, T. A. AND ROFF, D. A. 1989. Geographic variability in the incidence and heritability of wing dimorphism in the striped ground cricket, Allonemobius fasciatus. Heredity, 62, 315-318.

PRESS, W. H., FLANNERY, B. P., TEAKOLSKY, S. A. AND VetTerling, W. T. 1986. Numerical Recipes. Cambridge University Press, Cambridge.

ROFF, D. A. 1977. Dispersal in dipterans: its costs and consequences. J. Anim. Ecol., 46, 443-456.

ROFF, D. A. 1984. The cost of being able to fly: a study of wing polymorphism in two species of crickets. Oecologia, 63, 30-37.

ROFF, D. A. 1986a. The evolution of wing dimorphism in insects. Evolution, 40, 1009-1020.

ROFF, D. A. 1986b. The genetic basis of wing dimorphism in the sand cricket, Gryllus firmus and its relevance to the evolution of wing dimorphisms in insects. Heredity, 57, 221-231.

ROFF, D. A. AND FAIRBAIRN, D. J. 1990. Wing dimorphisms and the evolution of migratory polymorphisms among the Insecta. Am. Zool. (In press).

SOUTHWOOD, T. R. E. 1962. Migration of terrestrial arthropods in relation to habitat. Biol. Rev., 37, 171-214.

WALKER, T. J. 1987. Wing dimorphism in Gryllus rubens (Orthoptera: Cryllidae). Ann. ent. Soc. Am., 80, 547--560. 